\title{
VISCOUSLY DRIVEN PLASMA FLOWS IN THE DEEP GEOMAGNETIC TAIL
}

\author{
C.J. Owen and J.A. Slavin
}

Laboratory for Extraterrestrial Physics, NASA/Goddard Space Flight Center.

\begin{abstract}
We present an analysis, based on the principles of stress balance in a 1-dimensional current sheet, which considers the problem of closed magnetic flux transport into the deep tail by a "viscous"-like interaction between the solar wind and the magnetosphere. We illustrate our analysis with an example of ISEE-3 data showing strong tailward plasma sheet flows on apparently closed field lines in the deep tail. Apart from narrow regions adjacent to the magnetopause, these flows are not driven by the scattering of magnetosheath plasma into the magnetosphere. We estimate the fraction of the magnetosheath momentum flux needed to be anomalously transferred into the plasma sheet to drive the flows. In our example this is $\sim 6 \%$. No previously suggested mechanism (e.g., the Kelvin-Helmholtz Instability) has been shown capable of providing anomalous momentum transport of this magnitude. Our current understanding of the "viscous" interaction between the solar wind and magnetosphere is thus insufficient to explain these observations.
\end{abstract}

\section{Introduction}

Despite the clear dominance of reconnection processes in driving magnetospheric convection, both at the dayside magnetopause and in the magnetotail [e.g., Cowley, 1982], a number of studies have presented evidence of a "viscous"-like interaction between the solar-wind and the magnetosphere. For example, Eastman et al. [1976], Williams et al. [1985] and Mitchell et al. [1987] concluded that the presence of the lowlatitude boundary layer on closed field lines just inside the dayside magnetopause is evidence of such a process. Also, studies of the ISEE-3 deep tail data set [e.g., Slavin et al., 1987] show that under quiet geomagnetic conditions the plasma sheet magnetic field is generally northward, and the plasma flow antisunward across the entire tail. These conditions persist along the tail flanks even during disturbed times when southward magnetic fields arld high speed tailward flows are observed in the tail center [Slavin et al., 1985]. The northward plasma sheet fields indicate closed magnetospheric field lines being convected tailward against the field tension under the influence of a "viscous"-like interaction. Similar results have been reported by Heikkila [1988]. Also, Richardson et al. [1989] report tailward plasma flows which are too slow to be the reconnection driven flows generally associated with the distant tail plasma sheet. In this paper we present an analysis of "viscously-driven" plasma sheet flows based on the principles of stress balance in a 1-d current sheet. For illustration, we apply the analysis to an example of such flows from the ISEE-3 deep tail data set. We discuss the implications for previously suggested mechanisms of momentum transfer across the tail magnetopause.

\section{Stress Balance on Closed Plasma Sheet Field Lines}

In this section we discuss the balance of magnetic and plasma stresses in the plasma sheet from a theoretical viewpoint. We

\section{Copyright 1992 by the American Geophysical Union.}

Paper number 92GL01280

0094-8534/92/92GL-01280\$03.00 assume the plasma sheet is formed on either side of a $1-d$ current sheet in which the variations in parameters along the sheet are small compared to those across it, as appropriate in the deep tail where plasma pressure gradients are negligable, and that the plasma parameters are symmetrical on either side. Under these circumstances, and assuming a gyrotropic plasma, the stress balance conditions just outside the current sheet are

$$
\begin{gathered}
P_{\mathrm{II}}-P_{\perp}=\frac{B^{2}}{\mu_{0}} \\
P_{\perp}+\frac{B^{2}}{2 \mu_{0}}=\text { Constant }
\end{gathered}
$$

[Rich et al., 1972], where $P_{1}$ and $P_{\perp}$ are the parallel and perpendicular plasma pressures, and $B$ is the field strength.

The field and plasma configuration assumed in this paper is shown in Figure 1. The magnetic field is represented by heavy lines, and the plasma sheet is indicated by the shaded region. We assume the closed field lines threading the plasma sheet are being convected tailwards ( $-x$ direction) at some velocity $v_{F}$, while plasma flows into the sheet at speed $v_{\mathbf{N}}$, decelerates as it does work on the field, and emerges at speed $v_{\text {our }}$. In general, plasma flows into and out of the current sheet on both sides, although we show flow through the current sheet in one direction only for clarity. In this configuration the inflow and outflow velocities consist of a field aligned component plus an ExB drift away from the current sheet. We assume that the lobe field strength is greater than that in the plasma sheet, due to the diamagnetic effects in the latter, and that the lobe is devoid of plasma, such that $P_{\perp}=0$ in this region.

Figure 2 represents this field configuration and associated plasma flows in velocity space. Here, the unprimed frame $\left(V_{x}\right.$,

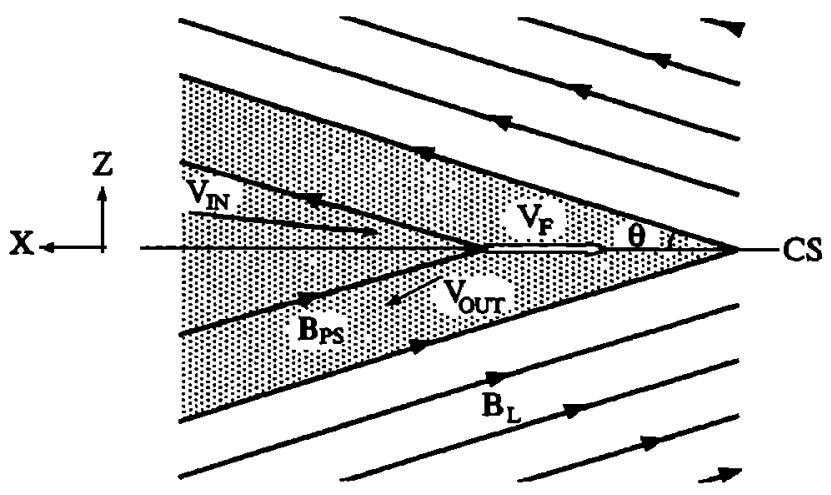

Fig. 1. The 1-D current sheet system used in this paper. The heavy arrowed lines represent the magnetic field, making an angle $\theta$ to the current sheet (labelled CS). The plasma sheet (shaded) is located on closed field lines which are being convected at. speed $V_{F}$ in the -ve $x$ direction. This convection is maintained by an inflow of plasma into the current sheet at speed $V_{\mathbb{N}}$, and a deceleration of the plasma resulting in an outflow at speed $V_{\text {our. }}$. The plasma sheet field strength is $B_{m}$ due to diamagnetic effects, while the lobe field strength is $B_{L-}$ 


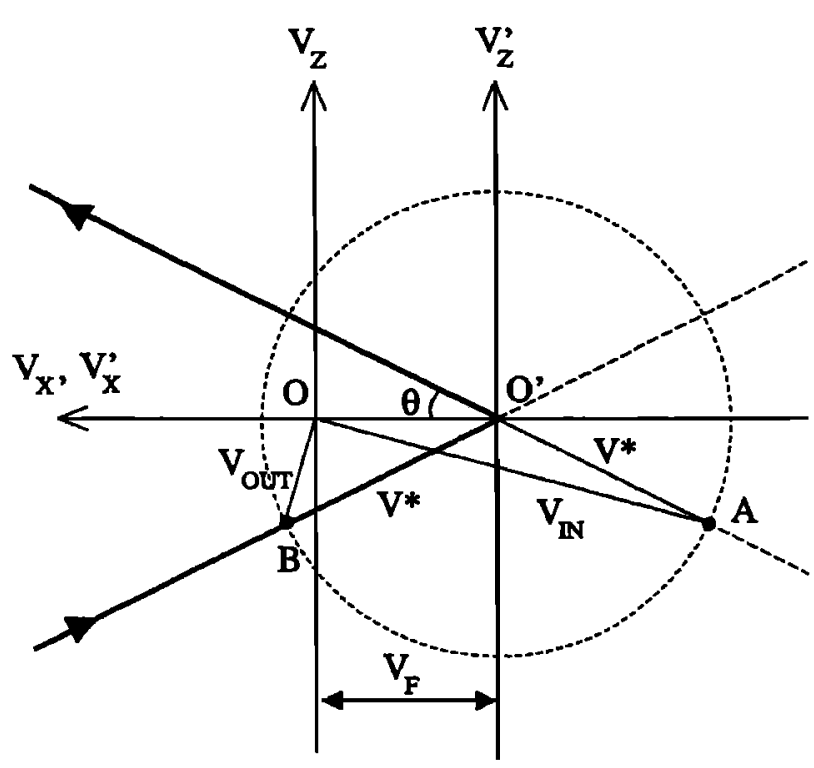

Fig. 2. The field and plasma flows in velocity space. The unprimed frame $\left(V_{x}, V_{z}\right)$ is the spacecraft rest frame, while in the primed frame $\left(V_{x}, V_{z}\right)$ the field line (solid arrowed line) is at rest. This frame moves at speed $V_{F}$ with respect to the spacecraft frame. In the primed frame the particles move into and out of the current sheet along the field direction at constant speed $\mathrm{V}^{*}$. The corresponding inflow and outflow speeds in the spacecraft frame are $V_{\mathrm{w}}$ and $\mathrm{V}_{\text {our }}$

$V_{\text {}}$ ) is the rest frame of the spacecraft, while the primed frame $\left(\mathrm{V}_{\mathrm{x}}{ }^{\prime}, \mathrm{V}_{\mathrm{z}}{ }^{\prime}\right)$ is that in which plasma sheet field lines (solid arrowed lines at an angle $\theta$ to the current sheet) are at rest. This frame moves at speed $V_{F}$ in the. $-V_{x}$ direction relative to the spacecraft frame, and is the frame in which the convection electric field is transformed away [deHoffman and Teller, 1950]. Hence in this frame the plasma inflow is simply field aligned at speed $\mathrm{V}^{*}$, as shown by the vector $\mathrm{O}^{\prime} \mathrm{A}$. Within the current sheet, the plasma is simply tumed around, and emerges as a field aligned beam of the same speed $V^{*}$ (vector $O^{\prime} B$ ). The corresponding inflow and outflow velocities in the unprimed frame are then given by $O A$ and $O B$ respectively.

Applying equation (1b) across the plasma sheet-lobe boundary, we obtain a value of $P_{\perp}$ in the plasma sheet given by

$$
P_{\perp}=\frac{\left(B_{L}^{2}-B_{P S}^{2}\right)}{2 \mu_{o}}
$$

Just outside the current sheet, where we assume the combined number density of the inflowing and outflowing plasma is $n_{p s}$, $P_{1}=n_{p s} m_{i} V^{* 2}$ in the field line rest frame $\left(m_{i}\right.$ is the mean ion mass). Substituting for $P_{1}$ and $P_{\perp}$ into equation (1a) we obtain

$$
V^{*}=\sqrt{\frac{\left(B_{L}^{2}+B_{P S}^{2}\right)}{2 n_{P S} m_{j} \mu_{o}}}
$$

Note that this velocity is fixed by the field strengths and plasma sheet density alone. By geometry we also obtain an expression for the inflow speed in the spacecraft frame

$$
V_{I N}=\sqrt{V_{F}^{2}+2 V_{F} V^{*} \cos \theta+V^{* 2}}
$$

With symmetrical inflow and outflow on each side of the current sheet, there is no net flow of plasma in the $\mathrm{x}$ - or $\mathrm{z}$ directions in the field line rest frame. Hence in the spacecraft frame the combined inflow and outflow simply gives a bulk

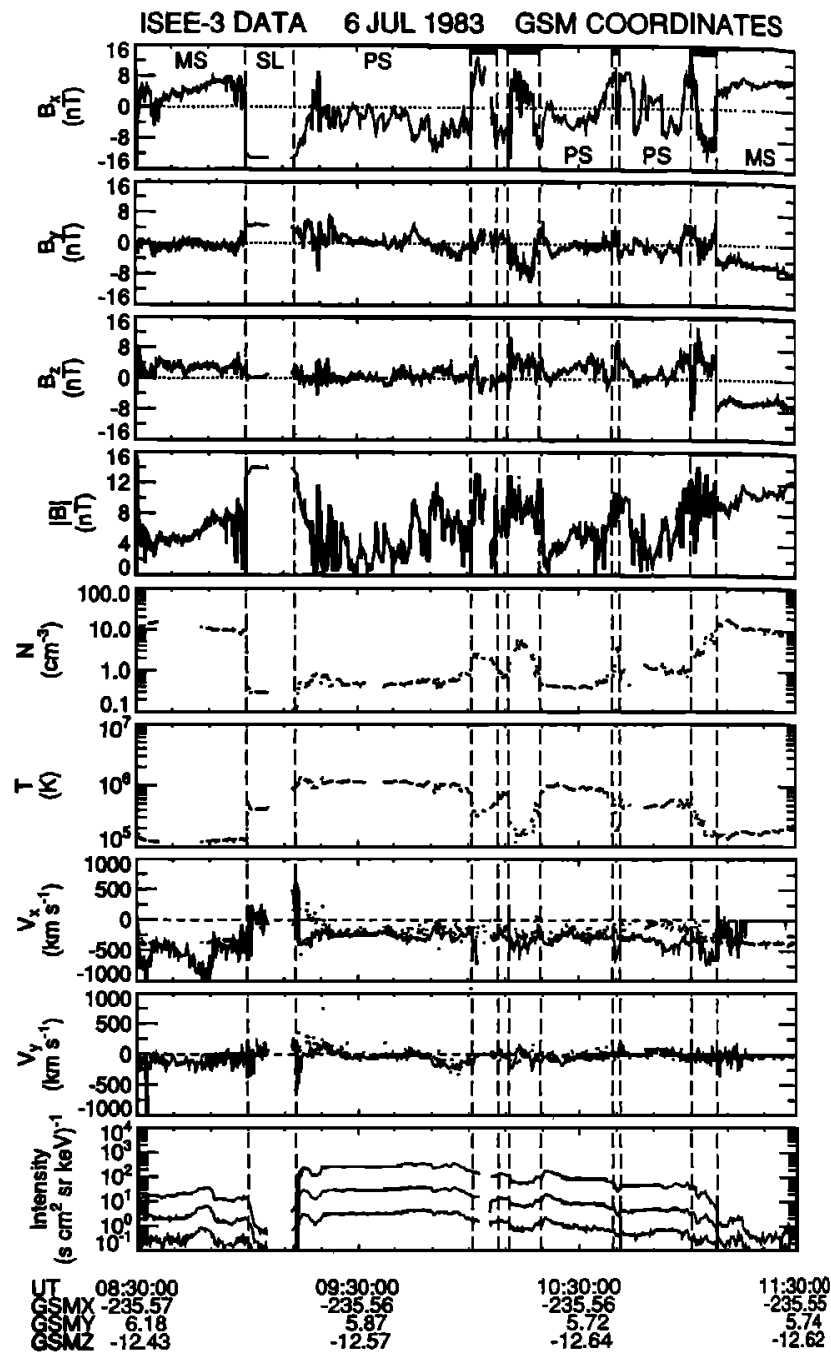

Fig. 3. ISEE-3 magnetic field, plasma and energetic ion data for July 6, 1983. ISEE-3 was located at $\mathrm{X}_{\mathrm{osm}} \sim 236 \mathrm{R}_{\mathrm{E}}$. From -0913 $--1100 \mathrm{UT}$, the field and plasma signatures indicate that ISEE3 is in the plasma sheet (PS). The predominantly +ve $B_{z}$ and the antisunward plasma flow indicates closed field lines being convected tailward against the field tension by a "viscous interaction" between the solar wind and the magnetosphere.

flow at the field line velocity $V_{F}$ in the $-x$ direction. This is the bulk velocity detected by the spacecraft instrumentation.

There are two popular generic mechanisms for the "viscous" transfer of solar wind momentum into the magnetosphere scattering of solar wind particles onto closed magnetospheric field lines, and anomalous momentum transport to existing magnetospheric plasma. We consider each mechanism in turn.

Mass Transfer. In this section, we calculate the amount of solar wind mass needed to be scattered into the plasma sheet to balance the observed field tension and drive the observed tailward flow. We assume that particles are instantaneously scattered onto closed magnetospheric field lines with no change of energy. Note also that in the tail $\left|B_{x}\right|>\left|B_{z}\right|$, so $\cos \theta-1$. Under these assumptions, we relate the value of $V_{F}$ in equation (4) to the observed plasma sheet speed $V_{P S}$, and the value of $V_{B V}$ with the observed sheath flow speed $V_{s H}$. Hence $V^{\prime}=V_{S H}-V_{P S}$ from equation (4), and using equation (3) we obtain a theoretical estimate for the plasma sheet density needed to provide the correct stress balance and plasma sheet flow 


$$
n_{P S}=\frac{\left(B_{L}^{2}+B_{P S}^{2}\right)}{2 m_{i} \mu_{o}\left(V_{S H}-V_{P S}\right)^{2}}
$$

This is a lower estimate of $n_{\mathrm{Ps}}$ since only that population scattered onto closed field lines from the magnetosheath is considered, and any pre-existing population already located on those field lines would increase this value.

Anomalous Momentum Transport. The second possibility for driving the "viscous"-like flows is that in which a fraction $\mathrm{K}$ of the sheath momentum flux is anomalously transferred to a preexisting population on closed plasma sheet field lines. It is outside the scope of this paper to review the various mechanisms suggested for this process (e.g., the Kelvin-Helmholtz instability (KHI)), but we can make an estimate of the fraction $\mathrm{K}$ required to provide the stress balance and drive the observed flows in the plasma sheet. We assume that the momentum flux is transferred to that part of the plasma sheet population (of density $n_{\mathrm{ps}} / 2$ ) moving into the current sheet. Hence

$$
K n_{S H} V_{S H}^{2}=\frac{1}{2} n_{P S} V_{I N}^{2}=\frac{1}{2} n_{P S}\left(V_{P S}+V^{*}\right)^{2}
$$

where we again put $\mathrm{V}_{\mathrm{F}}=\mathrm{V}_{\mathrm{PS}}$ and assumed $\cos \theta-1$. Hence

$$
K=\frac{n_{P S}}{2 n_{S H}} \cdot \frac{\left(V_{P S}+V^{*}\right)^{2}}{V_{S H}^{2}}
$$

where $V^{*}$ is given by equation (3). Again this is a minimum estimate for $\mathrm{K}$ since we have assumed that all of the transferred momentum is imparted as a parallel motion of the plasma sheet population (i.e. this population already possesses the correct $P_{\perp}$ to satisfy equation (2), and does not have to be heated in the perpendicular direction). We have also assumed that the momentum transferred is imparted only to the plasma flowing into the current sheet, and not to the outflowing plasma.

The above analyses can be used to determine the viability of the two processes in driving observed plasma flows in closed field line regions. In the next section we apply our analysis to an example of the observations which led to the statistical results of Slavin et al., [1985, 1987] and Heikkila [1988].

\section{Observations of Viscously Driven Plasma Sheet}

In this section we present observations made by ISEE-3, located at $x_{\text {OSM }}-236 R_{E}$, on July 6, 1983. Panels $1-4$ of Figure 3 show the GSM x, y and $z$ components and magnitude of the magnetic field measured by the JPL vector helium magnetometer. Panels 5-8 contain data from the Los Alamos electron plasma instrument and show the observed density, temperature, and GSM $x$ and $y$ components of the plasma flow (the $z$ component was not measured), plotted as individual points. The latter two panels also indicate (solid lines) the $x$ and $y$ components of the bulk flow determined from energetic ion measurements made by the Imperial College/Utrecht/ESTEC energetic particle anisotropy spectrometer (EPAS). This determination is valid only in the plasma sheet region [Daly et al., 1984] and assumes that the observed ion anisotropies are the result of isotropic distributions convecting with the thermal plasma flow. The final panel shows the omnidirectional energetic ion intensity in the lowest 3 energy channels (35-56, $56-91$ and 91-147 keV) of the EPAS instrument. The regions encountered by ISEE-3 are labelled in the top panel.

From the start of the interval until 0900 UT, ISEE-3 is located in the magnetosheath (labelled MS), observing cold, dense plasma, and magnetic fields which fluctuate rapidly in both strength and direction. Steady plasma flows (dots in panels 7 and 8) are observed, predominantly in the $-x$ GSM direction at
$-375 \mathrm{~km} \mathrm{~s}^{-1}$. At $0900 \mathrm{UT}$ the magnetic field strength rises rapidly, the density falls sharply, from $-10 \mathrm{~cm}^{-3}$ to $-0.3 \mathrm{~cm}^{-3}$, and the temperature rises from $1.2 \times 10^{5} \mathrm{~K}$ to $4 \times 10^{5} \mathrm{~K}$. Along with the strong magnetic field $(-14 \mathrm{nT})$, lying almost entirely in the $-x$ direction, this indicates that ISEE- 3 moves into the south lobe (SL) at this time. At -0913 UT, shortly after a data gap, the field strength falls, and although the plasma density increases to $-0.5 \mathrm{~cm}^{-3}$, this is well below that in the sheath. There is also an increase in temperature to $-1.2 \times 10^{6} \mathrm{~K}$. These signatures indicate that ISEE-3 enters the plasma sheet (labelled PS) at this time [e.g., Zwickl et al., 1984].

With the exception of 3 relatively brief intervals (1001-1008, 1011- 1019 and 1039-1041 UT), the spacecraft remains within the plasma sheet until $-1100 \mathrm{UT}$. During this time the field is depressed relative to the lobe, on average $-5 \mathrm{nT}$, and the $B_{z}$, although small $(-1-2 \mathrm{nT})$, is predominantly positive, i.e. northward. In the plasma sheet regions before $1040 \mathrm{UT}$, the plasma velocity from both the electron plasma instrument and the EPAS data indicate a tailward flow of $-250 \mathrm{~km} \mathrm{~s}^{-1}$. (The interval 1041-1100 UT will not be considered further here due to the large discrepancy between the ion and electron flow velocities). Note that these velocities remain well below those expected (e.g., Owen et al., 1991) or observed (e.g., Slavin et al., 1985) on reconnected open field lines $\left(-600 \mathrm{~km} \mathrm{~s}^{-1}\right)$. Also, the EPAS data in the final panel shows that the increase in ion flux at 0913 UT occurs almost concurrently with the depression in the field strength, and simultaneously in each energy channel. It thus appears that there is no dispersive separatrix layer at the lobe - plasma sheet interface. This feature would he exnecter on open, reconnected plasma sheet field lines, but not on closed field lines driven tailward against the field tension.

We also note 4 intervals, marked by the dark bar at the top of the first panel, in which the plasma density and energetic ion fluxes are intermediate between the levels observed in the plasma sheet and in the sheath. In these regions $B_{z}$ is for the most part strongly northward, and the electron flow speeds remain low. (The ions in these regions show strong streaming, as in the sheath, and analysis probably does not retum a reliable bulk flow velocity.) These intervals are consistent with a layer of closed field lines, lying adjacent to the magnetopause, which are also convecting tailward, and into which a significant amount of sheath plasma has gained entry. Finally, ISEE-3 returns to the magnetosheath at -1107 UT.

Both the plasma sheet intervals, at least before $1040 \mathrm{UT}$, and the 4 "entry layer" regions appear to be consistent with the statistical results of Slavin et al. [1985, 1987]. We concur with the conclusion of these authors that these flows result from closed field lines which are being dragged tailward by some form of "viscous" process. Hence we can now apply the analysis presented in the previous section to these data.

Mass Transfer. Using parameter values from the example above, $B_{L}-14 n T, B_{p s}-5 n T, V_{p s}-250 \mathrm{~km} \mathrm{~s}^{-1}$ (averaging the electron and ion velocities in the plasma sheet regions prior to $1040 \mathrm{UT}$ ), $\mathrm{V}_{\mathrm{sH}}-400 \mathrm{~km} \mathrm{~s}^{-1}$ (electrons only), and assuming a typical 4\% magnetosheath helium concentration (such that $\mathrm{m}_{1}=$ 1.12 proton masses), the plasma sheet density (equation 5) required to drive the flows by mass transfer is $n_{p s}-3 \mathrm{~cm}^{-3}$. This exceeds the density observed $\left(n_{\text {OBs }} \sim 0.5 \mathrm{~cm}^{-3}\right)$ by a factor 6. Hence, even with no pre-existing population, there is insufficient plasma on these field lines to drive the plasma flow by mass transfer. Indeed, if all of the observed plasma is scattered from the sheath, stress balance is satisfied by flux tubes moving tailward at only $50 \mathrm{~km} \mathrm{~s}^{-1}$ and we would expect a net tailward plasma sheet flow of the same speed. It thus appears, at least in this example, that mass diffusion across the magnetopause alone is insufficient to drive the observed plasma sheet flows.

However, the density observed during the 4 "entry layer" 
intervals (marked by the dark bars at the top of Figure 3) is $\geq$ $3 \mathrm{~cm}^{-3}$, such that the tailward flows in these regions may well be driven by mass transfer. Note that the last of these regions is located just inside the magnetopause, suggesting that mass transfer may only be important in narrow regions immediately adjacent to the magnetopause.

Anomalous Momentum Transport. We now calculate the value of $\mathrm{K}$ (equation 7 ) required to drive the observed plasma sheet flows. Using the observed parameters $\left(B_{L} \sim 14 n T, B_{P s}-\right.$ $5 \mathrm{nT}, \mathrm{n}_{\mathrm{Ps}}-0.5 \mathrm{~cm}^{-3}$, giving $\mathrm{V}^{*}-310 \mathrm{~km} \mathrm{~s}^{-1}$, and also $\mathrm{V}_{\mathrm{Ps}}-250$ $\mathrm{km} \mathrm{s}, \mathrm{V}_{\mathrm{sH}} \sim 375 \mathrm{~km} \mathrm{~s}^{-1}$, and $\mathrm{n}_{\mathrm{sH}} \sim 10 \mathrm{~cm}^{-3}$ ) we obtain $\mathrm{K}-$ 0.06 , i.e. $6 \%$ of the sheath momentum flux needs to be coupled into the plasma sheet to drive the observed flows. Note that the minimum momentum flux transfer required to drive the closed field lines tailward can be obtained by putting $\mathrm{V}_{\mathrm{ps}}=0$ in equation (7). In this case, $\mathrm{K}-0.02$, i.e. $2 \%$ of the sheath momentum flux is required to balance the magnetic stress so that the field lines are at rest in the spacecraft frame. If $K$ is less than this, the field lines must contract Earthward to satisfy stress balance. Using more typical parameter values (Slavin et al., [1985]) of $B_{L}-9.2 \mathrm{nT}, \mathrm{B}_{\mathrm{ps}}-4 \mathrm{nT}, \mathrm{n}_{\mathrm{ps}}-0.3 \mathrm{~cm}^{-3}$, and $\mathrm{n}_{\mathrm{sH}}$ $\sim 5 \mathrm{~cm}^{-3}$, we need $1.2 \%$ of the sheath momentum flux to be transferred in order to satisfy stress balance in the spacecraft frame, and larger values to drive tailward flows. Hence mechanisms postulated to explain the "viscous" interaction between the solar wind and the magnetosphere, and tailward plasma flows on closed field lines in the deep tail, must be capable of providing an anomalous momentum transport in excess of a few percent of the sheath momentum flux. Studies of the KHI [e.g., Miura, 1984] indicate that $0.6-2 \%$ of the sheath momentum flux may be routinely transferred across the magnetopause, and may thus be responsible for driving "viscous" flows under more typical conditions. A more recent study [Miura, 1992] has shown that $4 \%$ may be transferred under special circumstances, but the KHI has yet to be shown capable of driving flows of the magnitude presented here.

\section{Summary}

In this paper we have set up an analysis, based on the stress balance conditions for a 1-d current sheet, that addresses the problem of "viscous" transport of closed field lines into the deep tail. We have presented an example of tailward plasma sheet flow in the deep tail which appears to be located on closed magnetic field lines undergoing such transport. We have used our analysis to test whether these flows are consistent with the transfer of mass across the tail magnetopause and/or the anomalous transport of magnetosheath momentum. We find that the observed plasma sheet density is $-1 / 6$ of that required if scattering of magnetosheath plasma is responsible for driving the observed flows. However, we have also identified "entry layers" within the example in which the plasma density is consistent with the tailward flows being driven by mass transfer. Alternatively, some $6 \%$ of the sheath momentum flux must be transferred to the plasma sheet population if anomalous momentum transport is responsible. Although the KelvinHelmholtz Instability may provide a small tailward flow under certain parameter regimes, it is unlikely that it can drive flows of the magnitude presented here. We therefore conclude that our understanding of the "viscous" type interaction between the solar wind and the magnetosphere is far from complete.
Acknowledgement. We thank S.J. Bame (LANL), R.J. Hynds (IC) and E.J. Smith (JPL) for use of their ISEE-3 data. CJO acknowledges a NRC-NASA GSFC Research Associateship.

\section{References}

Cowley, S.W.H., The causes of convection in the Earth's magnetosphere: A review of developments during the IMS, Rev. Geophys. Space Phys., 20, 531, 1982.

Daly, P.W., et al., Survey of energetic ion anisotropies in the deep geomagnetic tail, J. Geophys. Res. 89, 10733, 1984.

deHoffman, F. and E. Teller, Magnetohydrodynamic shocks, Phys. Rev., 80, 692, 1950.

Eastman, T.E., et al., The magnetospheric boundary layer: Site of plasma, momentum and energy transfer from magnetosheath to magnetosphere, Geophys. Res. Lett., 3, 685, 1976.

Heikkila, W.J., Current sheet crossings in the distant magnetotail, Geophys. Res, Lett., 15, 299, 1988.

Mitchell, D.G., et al., An extended study of the low-latitude boundary layer on the dawn and dusk flanks of the magnetopause, J. Geophys. Res, 92, 7394, 1987.

Miura, A., Anomalous transport by magnetohydrodynamic Kelvin-Helmholtz instabilities in the solar wind-magnetosphere interaction, J. Geophys. Res., 89, 801, 1984.

Miura, A., Kelvin-Helmholtz instability at the magnetospheric boundary: Dependence on the magnetosheath sonic mach number, in press, J. Geophys. Res., 1992.

Owen, C.J., et al., Properties of the geotail plasma sheet Theory and observation, in Magnetospheric Substorms, ed. J.R. Kan et al., pp. 215-223, AGU, Washington, 1991.

Rich, F.J., et al.; On the balance of stresses in the plasma sheet, J. Geophys. Res., 77, 4670, 1972.

Richardson, I.G. and S.W.H. Cowley, Plasmoid associated energetic ion bursts in the deep geomagnetic tail: Properties of the boundary layer, J.Geophys.Res., 90, 12133, 1985.

Richardson, I.G., et al., ISEE-3 observations during the CDAW8 intervals: Case studies of the distant geomagnetic tail covering a wide range of geomagnetic activity, $J$. Geophys. Res., 94, 15189, 1989.

Slavin, J.A., et al., An ISEE-3 study of average and substorm conditions in the distant magnetotail, J. Geophys. Res., 90, 10875, 1985.

Slavin, J.A., et al. Magnetic configuration of the distant plasma sheet: ISEE-3 observations, Magnetotail Physics, ed. A.T.Y. Lui, pp. 59-63, JHU Press, Baltimore, 1987.

Williams, DJ., et al., Energetic particle observations in the lowlatitude boundary layer, J.Geophys. Res., 90, 5097, 1985.

Zwickl, R.D., et al., Evolution of the Earth's distant magnetotail: ISEE-3 electron plasma results, J. Geophys. Res., 89, $11007,1984$.

C.J. Owen and J.A. Slavin, Code 696, NASA/GSFC, Greenbelt, MD 20771, USA.

(Received: November 25, 1991; revised: April 24, 1992; accepted: May 20, 1992) 